\title{
Rodent Models and Behavioral Outcomes of Cervical Spinal Cord Injury
}

\section{Sydney A Geissler ${ }^{1}$, Christine E Schmidt ${ }^{1,2}$ and Timothy Schallert ${ }^{3,4 *}$}

${ }^{1}$ Department of Biomedical Engineering, The University of Texas, Austin, TX, USA

${ }^{2}$ Professor, J Crayton Pruitt Family Department of Biomedical Engineering, University of Florida, Gainesville, FL, USA

${ }^{3}$ Professor, Department of Psychology, The University of Texas, Austin, TX, USA

${ }^{4}$ Professor, Department of Neuroscience, The University of Texas, Austin, TX, USA

\begin{abstract}
Rodent spinal cord injury (SCI) models have been developed to examine functional and physiological deficits after spinal cord injury with the hope that these models will elucidate information about human SCI. Models are needed to examine possible treatments and to understand histopathology after SCl; however, they should be considered carefully and chosen based on the goals of the study being performed. Contusion, compression, transection, and other models exist and have the potential to reveal important information about $\mathrm{SCl}$ that may be related to human $\mathrm{SCl}$ and the outcomes of treatment and timing of intervention.
\end{abstract}

Keywords: Cervical spinal cord injury; Behavioral assessment; Rodent models; Regeneration; Spinal cord injury surgical models; Behavioral deficit; Functional recovery; Forelimb function

\section{Introduction}

Rodent models are designed to help predict functional outcomes of neurological disorders and injuries. Many of the behavioral outcomes appear to parallel clinical symptoms observed in human patients to a remarkable degree. About 11,000 people in the United States sustain SCI, leaving over 200,000 people living with debilitating levels of chronic paralysis. Further, $62 \%$ of these injuries are at the cervical level (http://www.uab.edu/medicine/sci/). Unilateral cervical spinal rodent models are desperately needed to assess the potential of treatments to help patients regain function. Using different injury models and sensitive behavioral tests that target the site and extent of injury, treatments aimed at improving functional recovery can be more adequately evaluated. Some models are considered more clinically relevant than others; however, it is important to remember that human SCI is very complex and many different injury types occur in human patients.

Understanding the strengths and limitations of the models will allow more relevant analysis of the injury, behavioral sequel, and therapeutic approaches.

\section{Injury Models of SCI}

Contusion and compression injuries are the most common forms of SCI in humans. Researchers can create these injury types using balloon compression, spring-loaded clips, and computer-controlled impact. These devices can be controlled for force of impact and dwell time that the force is applied. Each model has strengths and weaknesses (Table 1). This allows researchers to tailor the injury to their specific goals.

Balloon compression models are advantageous because they can precisely control the time and force of the compression and can be performed percutaneously, without a laminectomy. This model mimics a burst intervertebral disc injury in humans. These injuries are often not considered relevant because the decompression occurs much earlier than would occur in clinical cases. Spring-loaded clip models also allow good control of the time and force of the injury and are easy to perform with precise placement. This is one of the most inexpensive options for compression or contusion injury models. However, clip compression models require extensive decompression of the spinal canal which may cause further damage or inflammation. Compression injuries can also be performed with modified surgical forceps to create a reproducible functional deficit, and are more commonly used in a thoracic injury model $[1,2]$. Static weight compression is another model in which a known weight is placed gently on the spinal cord and held there for a controlled period of time [3]; because there is less acute damage, this model is not as clinically comparable, but still serves an important role to assess the damage of compression alone. These models are especially useful to assess the most optimal decompression time after injury [4] and have helped change the treatment of human patients after SCI. Indeed, prior to 1997 surgery was not performed as quickly as possible on patients in fear of doing further harm, but currently, patients are rushed into surgery to remove pressure from the spinal cord to prevent further damage [5].

Computer-controlled impactor injury models are generally recognized as the most clinically relevant models [6], and are well suited for measuring functional deficit. There are many different devices available for this model: (1) the MASCIC device uses a weight drop technique with different heights to control the severity of the lesion and has been well characterized for thoracic SCI [7], (2) the OSU device controls the displacement of the head to control severity of the lesion $[8,9],(3)$ the PinPoint device from Hatteras that allows control over velocity and displacement which was originally described for thoracic injury [10], and (4) the Infinite Horizon Impactor controls applied force to control the severity of the lesion, which has been thoroughly characterized for thoracic [11] and cervical SCI [12]. These approaches can all be used to create reproducible lesions; however, the necessary equipment can be expensive to purchase and the techniques require extensive training for proficiency. These models have been used to examine functional deficits and correlate them to histopathology [12], to examine demyelination [13], and as a means to assess biomaterial delivery [14] and cell delivery [15] for repair and regeneration.

Transection models are considered less clinically relevant; however, they allow researchers to be precise in the tracts affected, and allow them

*Corresponding author: Timothy Schallert, Department of Psychology and Department of Neuroscience, The University of Texas, Austin, USA, Tel: 512-423 1150; Fax: 512-232-4335; E-mail: tschallert@austin.utexas.edu

Received June 17, 2013; Accepted July 25, 2013; Published July 27, 2013

Citation: Geissler SA, Schmidt CE, Schallert T (2013) Rodent Models and Behavioral Outcomes of Cervical Spinal Cord Injury. J Spine S4: 001 doi:10.4172/2165-7939.S4-001

Copyright: (C) 2013 Geissler SA, et al. This is an open-access article distributed under the terms of the Creative Commons Attribution License, which permits unrestricted use, distribution, and reproduction in any medium, provided the original author and source are credited. 
Citation: Geissler SA, Schmidt CE, Schallert T (2013) Rodent Models and Behavioral Outcomes of Cervical Spinal Cord Injury. J Spine S4: 001. doi:10.4172/2165-7939.S4-001

Page 2 of 5

\begin{tabular}{|c|c|c|c|}
\hline Injury Model & Pros & Cons & Clinical Correlates \\
\hline \multicolumn{4}{|l|}{ Compression } \\
\hline $\begin{array}{ll}\text { - } & \text { Balloon } \\
\text { - } & \text { Spring-loaded clip } \\
\text { - } & \text { Modified forceps } \\
\text { - } & \text { Static weight }\end{array}$ & $\begin{array}{l}\text { - } \quad \text { Control of time of application } \\
\text { - } \quad \text { Control of force of application } \\
\text { - } \quad \text { Useful to assess decompression } \\
\text { time } \\
\text { - Inexpensive }\end{array}$ & $\begin{array}{l}\text { - Decompression occurs much earlier } \\
\text { than in clinical settings } \\
\text { - Extensive lamina removal causing } \\
\text { extensive decompression }\end{array}$ & $\begin{array}{ll}\text { - } & \text { Intervertebral disc burst injury } \\
\text { - } & \text { Determine optimal } \\
\text { decompression time }\end{array}$ \\
\hline \multicolumn{4}{|l|}{$\begin{array}{l}\text { Computer-controlled } \\
\text { impactor contusion }\end{array}$} \\
\hline $\begin{array}{ll}\text { - } & \text { MASCIC } \\
- & \text { OSU } \\
\text { - } & \text { Infinite Horizon } \\
\text { - } & \text { PinPoint }\end{array}$ & $\begin{array}{l}\text { - } \quad \text { Reproducible lesions } \\
\text { - } \quad \text { Useful to assess demyelination } \\
\text { and axon sparing } \\
\text { - } \quad \text { Considered most clinically } \\
\text { relevant model }\end{array}$ & - $\quad$ Expensive & $\begin{array}{l}\text { - } \quad \text { Considered most clinically } \\
\text { relevant model }\end{array}$ \\
\hline \multicolumn{4}{|l|}{ Transection } \\
\hline $\begin{array}{ll}\text { - } & \text { Dorsal hemisection } \\
\text { - } & \text { Dorsal quadrant } \\
\text { - } & \text { Lateral hemisection } \\
\text { - } & \text { Dorsal column }\end{array}$ & $\begin{array}{l}\text { - Useful to assess axonal } \\
\text { regeneration } \\
\text { - Inexpensive }\end{array}$ & - Considered less clinically relevant & $\begin{array}{l}\text { - } \quad \text { Stab or shooting injury } \\
\text { - } \quad \text { Brown-Sequard syndrome }\end{array}$ \\
\hline
\end{tabular}

Table 1: Pros and cons of surgical models. Differences in surgical models allow researchers to choose the surgical model to best study their goals.

to tailor their behavioral tests to assess the functional recovery most accurately. Transection models are usually performed with precision to remove specific tracts or areas of tissue. Dorsal hemisection [16], dorsal quadrant [17], lateral hemisection [18], dorsal column transection [16], and other models have been performed. These models are especially useful for assessing axon regeneration because the amount of axon sparing should be minimal or controllable, especially if a complete transection is performed. Researchers have used transection models to examine multiple treatments, including biomaterials [19], drugs or growth factors [20], and stem cell transplantation [21]. If researchers wanted to compare and assess the ability of various treatment options to support regeneration of the rubrospinal tract, then a quadrant or lateral hemisection can be very useful to guarantee that no axon sparing occurs. SCI lateral hemisection models include a lesion that removes half of the tissue laterally and is generally performed manually by the experimenter. This model has similar deficits as the human BrownSequard syndrome, in which half of the body loses function, whereas the other half of the body retains function [22]. Dislocation [23], and ischemia [24], has also been modeled anatomically and behaviorally.

\section{Behavioral Tests}

Researchers also need to determine which behavioral tests are most relevant for their species, model, and goals. Just as models will show different deficits based on which tracts are affected, behavioral tests reveal specific functional deficits? For example, if a model damages the corticospinal tract only, the animal's locomotion should not be seriously affected, so behavioral tests examining locomotion should not be the only ones used. Similarly, physical therapists and physicians examine human patients using the American Spinal Injury Association (ASIA) scale to look at specific deficits. This scale is used to determine the extent and level of injury for patients (http://www. asia-spinalinjury.org/). Patients are rated on specific functions that are associated with levels of the spinal cord (e.g., C5 is associated with bicep strength) and given a score of $0-5$ where 0 is complete paralysis, and 5 is normal function. Scores of all of the tested functions are then combined to give them an overall score from A-E where A is complete paralysis (including sensory function) and $\mathrm{E}$ is normal function (http:// www.asia-spinalinjury.org/elearning/ISNCSCI_Exam_Sheet_r4.pdf).

Vibrissae-elicited placing is a commonly used test to examine the sensorimotor function after spinal cord injury in rats $[18,25]$.
This test involves stimulating the whiskers on a table edge to elicit a motor response of the animal placing its forepaw onto the table to gain stability (http://homepage.psy.utexas.edu/homepage/group/ schallertlab/Et-1\%20placing.mpg). A graded score of 0-4 is assigned based on the extent of movement of the limb being tested where 0 is complete paralysis, and 4 is normal function. This test can indicate the integrity of motor and sensory tracts. This test eliminates the possibility of a reflex response being elicited; therefore, this test can reveal that a motor tract is intact along the distance it travels. Based on the level of function, this test may also reveal the extent of damage to the crosssectional area of the motor tract.

Contact placing is similar to vibrissae-elicited placing and involves a light touch to the paw from which the motion is being elicited; this test has been performed on rats [26] but is difficult to perform on mice. This involves a light touch that does not signal the pressure sensory nerves in the skin to elicit a response, and the experimenter must be careful to not move the limb or any joints during testing. This test can show if there is a reflex; however, it will not differentiate between a reflex and a complete tract connection, where the tactile information made it to the brain prior to eliciting the response. This test is similar to the tests performed for the ASIA scale used for SCI patients to determine if they can feel light touch. In human patients, they are asked to tell the doctor if they feel the light touch; however, with animals, that is not possible, so this test incorporates motor movement as the signal to show that the light touch is perceived.

Proprioceptive placing indicates whether the reflex arc is still present. If the animals are able to respond to light touch, this test will not work because they will respond before there is motion of the limb. The test has been performed on rats [26] but is difficult to perform in mice. By stretching the tendon as a joint is extended, a reflex response is elicited. This is similar to the reflex tests performed for the ASIA test in human SCI patients.

Limb use asymmetry during vertical-lateral exploration in a cylinder $(20 \mathrm{~cm}$ diameter, $30 \mathrm{~cm}$ height for rats; $11 \mathrm{~cm}$ diameter, 20 $\mathrm{cm}$ height for mice) is an easy, inexpensive test to determine whether the animal has a preference in its limb use; this test has been used extensively in rodents [25,27-30]. The cylinder test involves allowing the animals to move naturally and vertically explore the walls around them while the experimenter records which limbs are used for 
weight supported stepping on the wall (http://homepage.psy.utexas. edu/homepage/group/schallertlab/CylinderTest.mpg). This allows a clear determination of sidedness in natural function. This would allow experimenters to determine which side of the animal was more affected by the injury, and in the case of unilateral injuries, it allows the experimenters to determine the level of use of the ipsilesional vs. contralesional limb.

Swim tests can reveal functional deficits in forelimbs and hindlimbs. Normal rodent behavior when swimming to an escape platform is to hold both forelimbs forward under their chin in a planing position while stroking with the hindlimbs [31]. With a deficit in the forelimbs, an animal will not be able to plane their forepaws. This can result in one limb dragging. This test is very reliable for cervical SCI injuries to determine presence of forepaw planing behavior, the angle of a limb that is not planing, and deficits in hindlimb stroking motions [30].

Open-field locomotion is used to assess the animal during normal functional movement. This involves observing and analyzing the position and weight support on individual forelimbs during uninterrupted locomotion. These tests are sensitive to the amount of limb use when the animals are behaving naturally. One well standardized open field test is the Basso, Beattie, and Bresnahan (BBB) test. Although, it is better suited for thoracic injuries, it has been used in low cervical injuries extensively as well [32]. There are a few open-field locomotion tests, including the IBB [33], FLS [34], FLAS [35], and one developed by a group at the University of Provence in Marseille, France [36] that are more specifically designed for cervical injuries. Each test provides a score for different behaviors that are sequential categories of forelimb function, including weight support on the limbs, paw position and orientation, and swinging motion. These tests observe animals in a similar situation to human locomotion because they do not use forced motion of the ipsilesional limb and allow the animal to compensate for the injury the way they usually compensate while moving on their own. Compensation occurs frequently in rodents and humans to increase speed, efficiency, or accuracy of a movement but does not exercise or demonstrate the functional ability of the ipsilesional limb.

Forepaw dexterity tests show fine motor movements and reveal paw strength and function. Animals can use their paws differently based on the model and the tracts affected. Similarly to human deficits, some animals cannot grip an object, and some cannot release their grip on an object. Some can move individual digits while others have only moderate wrist or shoulder function. A few pasta tests have been developed to reveal these differences. The Jones and Whishaw groups have developed a test to examine individual digit function $[37,38]$, while Schmidt group uses a similar pasta type to examine overall forelimb function [18]. Other handling tests use different types of foods, including macaroni pasta, peanuts, and grapes among other types [39] to examine functional deficits. Time to eat the object, forepaw use, and individual digit motion are all examined in these tests. Improvement can be quantified as the animals increase use of the ipsilesional limb. These are similar to human SCI patients' abilities to grip and manipulate objects. The ASIA exam includes finger strength and abduction assessment to determine injury level (http://www.asiaspinalinjury.org/).

Forced motion tests have been developed to assess the functional abilities of each forelimb while preventing compensation with the other limb. The postural instability test (PIT) (http://homepage.psy. utexas.edu/homepage/group/schallertlab/PIT1.mpg) and a forelimb alternation test (http://homepage.psy.utexas.edu/homepage/group/ schallertlab/alt.v.nonalt_mpg.mpg) have been used to examine forelimb function during forced forward motion [18]. These tests are scored by determining the distance before motion is elicited while the animal is moved forward with only forelimb support, showing improvement as the score aligns with the pre-op distance. These tests allow researchers to examine an animal's ability to use the limbs while removing the chance for compensation with the other limb, giving the experimenter an understanding of actual functional abilities whether or not the animal has been compensating for that same motion. This is more comparable to clinical tests, as most of the tests performed on patients examine a patient's ability when they consciously attempt to make individual movements.

This is by no means a comprehensive review, and many other behavioral tests exist such as the Montoya staircase food-retrieval [12, $25]$, grooming [12,27], automated walkway [12,27], inclined plane [32,40-42], and horizontal ladder [27] tests. Each of these tests can reveal specific functional recovery and researchers should consider which tests are most relevant for their goals and expectations. Further, we did not describe many sensory tests here and these should also be considered. The dot patch removal test [16,25,30], Von Frey hairs [42], and heat test [43] can all elicit responses to sensory input and may provide insight to hyperalgesia [44].

\section{Considerations}

When determining which animal model and behavioral tests to use, researchers have many factors to consider, including goals, speciesspecific differences, cost, and analysis time and ability. The detail that can be attained using many behavioral tests should not outweigh the importance of standardized measurement between research groups with similar goals. Comparability of the models and assessment is critical to translate treatment to the clinic. Further, it is important to recognize the importance of planning. Researchers should choose models to meet their goals rather than performing a barrage of tests and reporting the most significant findings.

The therapeutic goals should match the damage expected by the model [7]. For example, if a treatment is designed to specifically connect rubrospinal tract neurons, a moderate midline contusion injury will not suffice to cause much damage to that region. The region of interest also needs to be chosen wisely to achieve specific deficits and to allow for recovery.

To determine which species and strain to use for the study, researchers must also consider what their goals are. Rats exhibit similar physiological changes to humans after SCI in that a cavity forms within the area. Mice do not exhibit this physiology; however, genetically controlling mice is much easier and physiological changes can be examined more easily using specific mouse strains. Such differences in rodents can provide powerful analysis if the correct animal model is chosen. Researchers have also used larger animals such as cats [44,45], dogs [46], and primates [47], or smaller animals, such as eels [48], zebrafish [49], and lamprey [50]. The regenerative capacity in each species varies and provides a platform for examining different characteristics of injury based on the experiment.

With each of these considerations also comes cost. Different injury models may be much more expensive to implement. The Infinite Horizon Impact or is much more expensive than a set of aneurysm clips, and a transection model can be performed with standard surgical instruments, making it even less expensive. Further, the cost of the species used, and the cost of post-operative animal care should be considered.

Anatomical analysis of the histopathology can be time 
consuming. Tract tracing can elucidate which specific tracts were spared or regenerated after injury, and specific staining can inform researchers about myelin damage and glial responses. However, physiological examination does not speak to the function of the tissue. Electrophysiological assessment would be very valuable to determine functionality of specific regions; however, it is very difficult to carry out. Behavioral assessment should be performed to assess functional deficit and recovery in combination with histological assessment. Behavioral assessment can elucidate the influence of the damage and repair of the tissue on overall function. Different models can make some analysis more easily performed or relevant; for example, transection models can be advantageous to eliminate axon sparing, but sprouting and axon sparing are more relevant in a contusion model. Transection models create a space for placement of pre-formed biomaterials, but if a treatment is injectable, a contusion model may be chosen to more closely mimic clinical injuries.

It is important to consider the leading causes of morbidity and mortality among rodent test subjects. Autophagia is common for all injury models $[51,52]$. Respiratory tract infections and decubitus are also concerns for researchers working with cervical spinal cord injury [53]. Urinary tract infections are less prevalent with cervical than with thoracic SCI models but should still be monitored [53].

\section{Conclusion}

Rodent models cannot predict exactly how human patients respond to SCI physiologically or functionally; however, they provide an early assessment platform for treatment and physiological damage after SCI. Human SCI is very complex and there is an appreciation among researchers that the models cannot mimic the injuries perfectly, nor can they expect all behavioral symptoms observed in humans to have correlates in animals, or vice versa. It is important to examine behavioral deficits in rodents, even without a human correlate, to determine if there is a functional change. Although humans may not show an obvious vibrissae-elicited placing reaction, this test can reveal a loss, and a return, of sensory and motor communication between the brain and spinal cord. An animal's ability to perform in different tasks can reveal specific tracts that are regenerating, even if they are not in the same location in the human spinal cord. This can reveal a promising treatment that can then be tested further on higher species or directly on humans in a hope to help with recovery. It must be kept in mind that treatments that rescue neurons from degeneration may sometimes have an adverse effect on functional outcome [54].

Many surgical models exist to study SCI in rodents; however, it is important to assess the goals and expectations of the experiment before choosing a model. Similarly, behavioral tests need to be chosen carefully to ensure that a researcher is examining a relevant function. It can be tempting to use a barrage of tests and use the tests that show the most promising or significant results; however, this is not a scientifically sound way to determine the recovery after SCI. Each aspect of a study should be planned before the experiment is begun and a pilot study is recommended to provide insight into what is expected from each model and treatment to minimize animal use and unnecessary experimental time during the experiment.

\section{Acknowledgements}

This work was supported in part by the National Institutes of Health (CES) and the National Science Foundation Graduate Research Fellowship Program (SAG). NIH (Grant \# R21 NS074162), NSF GRFP Fellowship (Grant \# 2011112479).

\section{Author Disclosure Statement}

There are no competing financial interests.

\section{References}

1. Borgens RB, Shi R (2000) Immediate recovery from spinal cord injury through molecular repair of nerve membranes with polyethylene glycol. FASEB J 14:2735.

2. Borgens RB (2001) Cellular Engineering: molecular repair of membranes to rescue cells of the damaged nervous system. Neurosurgery 49:370-379.

3. Moradi F, Bahktiari M, Joghataei MT, Nobakht M, Soleimani M et al. (2012) BD PuraMatrix peptide hydrogel as a culture system for human fetal Schwann cells in spinal cord regeneration. J Neurosci Res 90:2335-2348.

4. Carlson GD, Minato Y, Okada A, Gorden CD, Warden KE et al. (1997) Early time-dependent decompression for spinal cord injury: vascular mechanisms of recovery. J Neurotrauma 14: 951-962.

5. Rahimi-Movaghar V (2005) Efficacy of Surgical Decompression in the setting of complete thoracic spinal cord injury. J Spinal Cord Med 28: 415-420.

6. Talac R, Friedman JA, Moore MJ, Lu L, Jabbari E et al. (2004) Animal models of spinal cord injury for evaluation of tissue engineering treatment strategies. Biomaterials 25: 1505-1510.

7. Agrawal G, Kerr C, Thakor NV, All AH (2010) Characterization of graded MASCIS contusion spinal cord injury using somatosensory evoked potentials. Spine 35: 1122-1127.

8. Stokes BT (1992) Experimental spinal cord injury: a dynamic and verifiable injury device. J Neurotrauma 9: 129-131.

9. Stokes BT, Noyes DH and Behrmann DL (1992) An electromechanical spinal injury technique with dynamic sensitivity. J Neurotrauma 9: 187-195.

10. Bilgen M (2005) A new device for experimental modeling of central nervous system injuries. Neurorehab Neural Repair 19: 219-226.

11. Scheff SW, Rabchevsky AG, Fugaccia I, Main JA, Lumpp JE Jr (2003) Experimental modeling of spinal cord injury: characterization of a force-defined injury device. J Neurotrauma 20: 179-193.

12. Streijger F, Beernink TM, Lee JH, Bhatnagar T, Park S et al. (2013) Characterization of a cervical spinal cord hemicontusion injury in mice using the infinite horizon impactor. J Neurotrauma 30: 869-883.

13. Schonberg DL, Goldstein EZ, Sahinkaya FR, Wei P, Popovich PG et al. (2012) Ferritin stimulates oligodendrocyte genesis in the adult spinal cord and can be transferred from macrophages to NG2 cells in vivo. J Neurosci 32: 5374-5384.

14. Lu P, Wang Y, Graham L, McHale K, Gao M et al. (2012) Long-distance growth and connectivity of neural stem cells after severe spinal cord injury. Cell 150 1264-1273.

15. White TE, Lane MA, Sandhu MS, O'Steen BE, Fuller DD et al. (2010) Neuronal progenitor transplantation and respiratory outcomes following upper cervical spinal cord injury in adult rats. Exp Neurol 225: 231-236.

16. Onifer SM, Zhang YP, Burke DA, Brooks DL, Decker JA et al. (2005) Adult rat forelimb dysfunction after dorsal cervical spinal cord injury. Exp Neurol 192: 25-38.

17. Houle JD, Tom VJ, Mayes D, Wagoner G, Phillips N et al. (2006) Combining an autologous peripheral nervous system "bridge" and matrix modification by chondroitinase allows robust, functional regeneration beyond a hemisection lesion of the adult rat spinal cord. J Neurosci 26: 7405-7415.

18. Khaing ZZ, Geissler SA, Jiang S, Milman BD, Aguilar SV et al. (2012) Assessing forelimb function after unilateral cervical spinal cord injury: novel forelimb tasks predict lesion severity and recovery. J Neurotrauma 29: 488-498.

19. Johnson PJ, Parker SR, Sakiyama-Elbert SE (2010) Fibrin-based tissue engineering scaffolds enhance neural fiber sprouting and delay the accumulation of reactive astrocytes at the lesion in a subacute model of spinal cord injury. J Biomed Mater Res A 92: 152-163.

20. Taylor SJ, Sakiyama-Elbert SE (2006) Effect of Controlled delivery of neurotrophin-3 from fibrin on spinal cord injury in a long term model. J Control Release 116: 204-210.

21. Johnson PJ, Tatara A, McCreedy DA, Shiu A, Sakiyama-Elbert SE (2010) Tissue-engineered fibrin scaffolds containing neural progenitors enhance functional recovery in a subacute model of SCI. Soft Matter 6: 5127-5137.

22. Brown-Sequard CE (1868) Lectures on the physiology and pathology of the nervous system; and on the treatment of organic nervous affections. Lance 92: 593-596. 
Citation: Geissler SA, Schmidt CE, Schallert T (2013) Rodent Models and Behavioral Outcomes of Cervical Spinal Cord Injury. J Spine S4: 001. doi:10.4172/2165-7939.S4-001

Page 5 of 5

23. Fice JB, Cronin DS, Panzer MB (2011) Cervical spine model to predict capsular ligament response in rear impact. Ann Biomed Eng 39: 2152-2162.

24. Xu XJ, Hao JX, Aldskogius H, Seiger A, Wiesenfeld-Hallin Z (1992) Chronic pain-related syndrome in rats after ischemic spinal cord lesion: a possible animal model for pain in patients with spinal cord injury. Pain 48: 279-290.

25. Schallert T, Fleming SM, Leasure JL, Tillerson JL, Bland ST (2000) CNS plasticity and assessment of forelimb sensorimotor outcome in unilateral rat models of stroke, cortical ablation, parkinsonism and spinal cord injury. Neuropharmacology 39: 777-787.

26. Ramón-Cueto A, Cordero MI, Santos-Benito F, Avila J (2000) Functiona recovery of paraplegic rats and motor axon regeneration in their spinal cords by olfactory ensheathing glia. Neuron 25: 425-435.

27. Gensel JC, Tovar A, Hamers FP, Deibert RJ, Beattie MS et al. (2006) Behavioral and histological characterization of unilateral cervical spinal cord contusion injury in rats. J Neurotrauma 23: 36-54.

28. Liu Y, Kim D, Himes BT, Chow SY, Schallert T et al. (1999) Transplants of fiborblasts genetically modified to express BDNF promote regeneration of adult rat rubrospinal axons and recovery of forelimb function. J Neurosci 19: 43704387.

29. Schallert T, Woodlee MT and Fleming SM (2002) Disentangling multiple types of recovery from brain injury. J Krieglstein, S Klumpp (Eds), Pharmacology of Cerebral Ischemia, Stuttgart, Medpharm Scientific, 201-216.

30. Kim D, Schallert T, Liu Y, Browarak T, Nayeri N et al. (2001) Transplantation of genetically modified fibroblasts expressing BDNF in adult rats with subtotal hemisection improves specific motor and sensory functions. Neurorehabil Neural Repair 15: 141-150.

31. Gruner JA, Altman J (1980) Swimming in the rat: Analysis of locomotor performance in comparison to stepping. Exp Brain Res 40: 374-382.

32. Nguyen DH, Cho N, Satkunendrarajah K, Austin JW, Wang J et al. (2012) Immunoglobulin G (IgG) attenuates neuroinflammation and improves neurobehavioral recovery after cervical spinal cord injury. J Neuroinflammation 9: 224.

33. Irvine KA, Ferguson AR, Mitchell KD, Beattie SB, Beattie MS et al. (2010) A novel method for assessing proximal and distal forelimb function in the rat: the Irvine, Beatties and Bresnahan (IBB) Forelimb Scale. J Vis Exp 46: 2246.

34. Sandrow HR, Shumsky JS, Amin A, Houle JD (2008) Aspiration of a cervical spinal contusion injury in preparation for delayed peripheral nerve grafting does not impair forelimb behavior or axon regeneration. Exp Neurol 210: 489-500.

35. Anderson KD, Sharp KG, Hofstadter M, Irvine KA, Murray M et al. (2009) Forelimb locomotor assessment scale (FLAS): novel assessment of forelimb dysfunction after cervical spinal cord injury. Exp Neurol. 220: 23-33.

36. Martinez M, Brezun JM, Bonnier L, Xerri C (2009) A new rating scale for openfield evaluation of behavioral recovery after cervical spinal cord injury in rats. $J$ Neurotrauma 26: 1043-1053.

37. Allred RP, Adkins DL, Woodlee MT, Husbands LC, Maldonado MA et al. (2008) The vermicelli handling test: a simple quantitative measure of dexterous forepaw function in rats. J Neurosci Methods 170: 229-244.

38. Tennant KA, Asay AL, Allred RP, Ozburn AR, Kleim JA et al. (2010) The vermicelli and capellini handling tests: simple quantitative measures of dexterous forepaw function in rats and mice. J Vis Exp 41: 2076.

39. Whishaw IQ, Coles BLK (1996) Varieties of paw and digit movement during spontaneous food handling in rats: Postures, bimanual coordination, preferences, and the effect of forelimb cortex lesions. Behav Brain Res 77: 135-148.

40. Rivlin AS, Tator CH (1978) Effect of duration of acute spinal cord compression in a new acute cord injury model in the rat. Surg Neurol 10: 38-43.

41. Fehlings MG, Tator $\mathrm{CH}$ (1995) The relationships among the severity of spinal cord injury, residual neurological function, axon counts, and counts of retrogradely labeled neurons after experimental spinal cord injury. Exp Neurol 132: 220-228.

42. Anderson KD, Sharp KG, Steward O (2009) Bilateral cervical contusion spinal cord injury in rats. Exp Neurol 220: 9-22.

43. Detloff MR, Wade RE Jr, Houle JD (2013) Chronic at- and below-level pain after moderate unilateral cervical spinal cord contusion in rats. J Neurotrauma 30: 884-890.

44. Blight AR (1983) Cellular morphology of chronic spinal cord injury in the cat: Analysis of myelinated axons by line-sampling. J Neurosci 10: 521-543.

45. Goto T, Hoshino Y (2001) Electrophysiological, histological, and behavioral studies in a cat with acute compression of the spinal cord. J Orthop Sci 6: 59-67.

46. Nylander WA, Plunkett RJ, Hammon JW, Oldfield EH, Meacham WF (1982) Thiopental modification of ischemic spinal cord injury in the dog. AnnThorac Surg 33: 64-68.

47. Iwanami A, Kaneko S, Nakamura M, Kanemura Y, Mori H et al. (2005) Transplantation of human neural stem cells for spinal cord injury in primates. $J$ Neurosci Res 80: 182-190.

48. Doyle LM, Stafford PP, Roberts BI (2001) Recovery of locomotion correlated with axonal regeneration after a complete spinal transection in the eel. $J$ Neuroscience 107: 169-179.

49. Bhatt DH, Otto SJ, Depoister B, Fetcho JR (2004) Cyclic AMP-induced repair of zebrafish spinal circuits. Science 305: 254-258.

50. Oliphint PA, Alieva N, Foldes AE, Tytell ED, Lau BY et al. (2010) Regenerated synapses in lamprey spinal cord are sparse and small even after functional recovery from injury. J Comp Neurol 518: 2854-2872.

51. Lynskey JV, Sandhu FA, Dai HN, McAtee M, Slotkin JR et al. (2006) Delayed intervention with transplants and neurotrophic factors supports recovery of forelimb function after cervical spinal cord injury in adult rats. J Neurotrauma 23: 617-634.

52. Anderson KD, Sharp KG, Steward O (2009) Bilateral cervical contusion spinal cord injury in rats. Exp Neurol 220: 9-22.

53. Khan T, Havey RM, Sayers ST, Patwardhan A, King WW (1999) Animal models of spinal cord contusion injuries. Lab Anim Sci 49: 161-172.

54. Schallert T, Lindner M D (1990) Rescuing neurons from trans-synaptic degeneration after brain damage: Helpful, harmful or neutral in recovery of function. Can J Psychology 44: 276-292.
This article was originally published in a special issue, Brain \& Spinal Cord Injury handled by Editor. Dr. Chen Gaung Yu, University of Kentucky, USA 